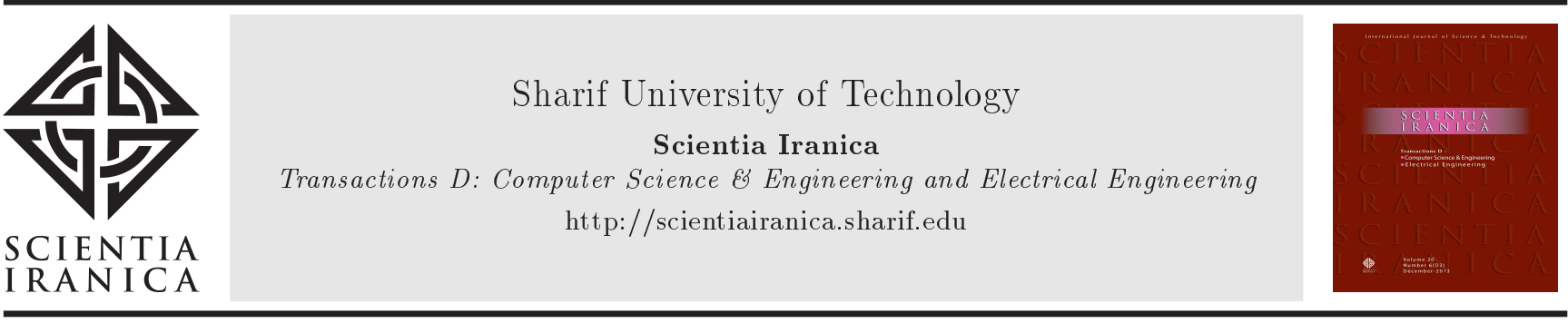

\title{
Quadrature oscillator based on a novel low-voltage ultra-low-power quasi-floating-gate DVCC
}

\author{
F. Khateb ${ }^{a, b, *}$, N. Khatib ${ }^{a}$, J. Koton ${ }^{c}$, and N. Herencsar ${ }^{c}$ \\ a. Department of Microelectronics, Brno University of Technology, Technicka 10, Brno, Czech Republic. \\ b. Faculty of Biomedical Engineering, Czech Technical University in Prague, nám. Sítná 3105, Kladno, Czech Republic. \\ c. Department of Telecommunications, Brno University of Technology, Purkynova 118, Brno, Czech Republic.
}

Received 1 April 2015; received in revised form 22 November 2016; accepted 2 January 2017

\section{KEYWORDS}

Quadrature oscillator; Quasi-floating-gate MOST;

Low-voltage ultra-lowpower analog circuit design;

DVCC.

\begin{abstract}
In this work, a new realization topology of the low-voltage ultra-low-power quadrature oscillator is presented. This quadrature oscillator utilizes only two active elements, namely, Differential Voltage Current Conveyors (DVCCs), and five passive ones. All of the elements are grounded, which is recommended for integrated circuit implementation. The DVCC is based on quasi-floating-gate MOS transistor, which is a distinct technique from the conventional one, featuring operation at low-voltage and ultra-low-power conditions; hence, the proposed DVCC works with low supply voltage of $\pm 400 \mathrm{mV}$ and consumes power of merely $6.6 \mu \mathrm{W}$. Thanks to these features, the total power dissipation of the oscillator is only $0.28 \mathrm{~mW}$. The simulation results using $0.18-\mu \mathrm{m}$ TSMC CMOS technology are included in order to prove validity of the design.
\end{abstract}

(C) 2018 Sharif University of Technology. All rights reserved.

\section{Introduction}

Evidently, among many parameters of great interests in electronic circuits performance improvement, LowVoltage (LV) Low-Power (LP) ones are probably the first priority when considering their contribution to prolonging battery life of portable devices. LV LP parameters are indispensable in a myriad of biomedical portable devices and wireless technology, e.g., cellular phones, laptop computers, etc. [1].

The formidable challenge in LV analog circuit design is the relatively high threshold voltage value of MOSFET transistor (MOST) in comparison with the used supply voltage. Of late, the threshold voltage value has been considered as an obstacle to the input

*. Corresponding author. Tel.: +420 541146128 E-mail addresses: khateb@feec.vutbr.cz (F. Khateb); khatib@feec.vutbr.cz (N. Khatib); koton@feec.vutbr.cz (J. Koton); herencsn@feec.vutbr.cz(N.Herencsar). signal that limits the input voltage range of the analog circuits. As a consequence, several design techniques have been proposed to overcome this limitation [2].

In addition to the conventional low-voltage ana$\log$ circuit design techniques, the Bulk-Driven (BD), Floating-Gate (FG), and Quasi-Floating-Gate (QFG) are by far the most popular unconventional techniques. These unconventional techniques can be used to avoid complexity of the circuit and achieve attractive results under the LV domain [2]. They have the ability to operate without concern for the threshold voltage. In other words, these techniques make the transistor able to operate with the supply voltage close to its threshold voltage.

Nevertheless, the challenge often encountered in these unconventional techniques is the small transconductance value compared with the conventional gatedriven one. This issue can be translated into many undesirable traits such as poor gain-bandwidth product, low frequency response, and low speed [2]. However, a multitude of circuits can exploit these disadvantages; biomedical, telemetry, real time speech recognition, 
and other systems are utilizing signals with low frequency response [3-7].

Recently, the bulk-driven and the floating-gate techniques have successfully been employed in many LV analog circuit designs [8,9]. However, there are some limitations and drawbacks to these techniques. With regard to the bulk-driven technique, its bulk transconductance is approximately 2.5 to 5 times lower than the conventional gate transconductance, which results in a lower unity gain frequency and higher input-referred noise; besides, a latch-up may occur, which limits the maximum operating voltage [10-16]. In respect of the floating-gate technique, it mainly suffers from the increased silicon area, initial charge trapped in the floating gates, and approximately 1.6 to 2 times lower value of the equivalent transconductance than the conventional gate transconductance, resulting in gain-bandwidth product degradation. It is interesting that these limitations and drawbacks could be eliminated by using the alternative QFG technique [1726].

The QFG principle is similar to the FG one with some variations; in case of $\mathrm{FG}$, unlike in QFG, the DC biasing point for the transistor is floating [27-32]. The non-floating DC biasing point in case of QFG is achieved by connecting the gate of the transistor to a suitable bias voltage via a large-value resistor $\left(R_{\mathrm{LARGE}}\right)$, as illustrated in Figure 1(a). The value of the bias voltage should be chosen so that the MOST is placed in the saturation region.

Obviously, applying the large-value resistor is not easy from the viewpoint of fabrication, since it increases the chip area of the system. However, to avoid the difficulty in design, stemming from the large resistor size, a MOST in the cut-off region (diode connected transistor) is used instead of the resistor, as shown in Figure 1(b) [33-35]. This very large voltage dependent resistor (diode connected transistor) can take values in the range of hundreds of Giga Ohms.

The equivalent circuit of QFG MOST is given in Figure 1(c). As shown in [35], the voltage transfer can be represented by:

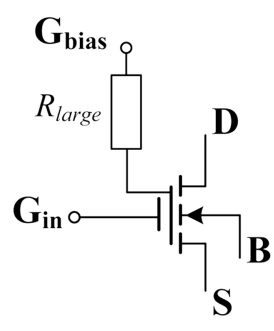

(a)

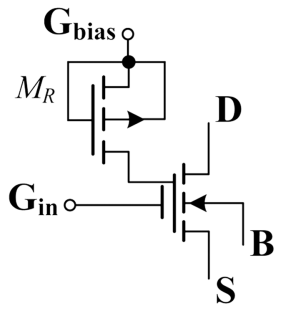

(b)

$$
\begin{aligned}
V_{G}= & \frac{s R_{\text {larg }}}{1+s R_{\text {larg }} C_{\text {total }}}\left(C_{i n} V_{i n}+C_{G D} V_{D}+C_{G S} V_{S}\right. \\
& \left.+C_{G B} V_{B}\right)
\end{aligned}
$$

where $C_{\text {total }}$ is the total capacitance, and is given by:

$$
C_{\text {total }}=C_{\text {in }}+C_{\mathrm{GD}}+C_{\mathrm{GS}}+C_{\mathrm{GB}}+C_{\mathrm{GD}}^{\prime} \text {, }
$$

where $C_{\mathrm{GD}}, C_{\mathrm{GS}}$, and $C_{\mathrm{GB}}$ denote the capacitances from gate to drain, to source, and to bulk, respectively. $C_{\mathrm{GD}}^{\prime}$ denotes the capacitances from gate to drain of the $M_{R}$ transistor.

The signal at the QFG of the MOST depends on the inputs signal $V_{i n}$ with the ratio of $C_{i n} / C_{\text {total }}$. Hence, the input signal is attenuated, which potentially enables rail-to-rail input swing $[35,36]$.

Based on the above mentioned advantages of the QFG MOST, this paper introduces a new design of DVCC utilizing the QFG technique. The low-voltage supply of $\pm 400 \mathrm{mV}$, ultra-low-power consumption of $6.6 \mu \mathrm{W}$, acceptable current, and voltage bandwidths of $19 \mathrm{MHz}$ are the major attractive characteristics of the circuit proposed. Furthermore, with the flexibility in design trade-offs between LV LP and an acceptable bandwidth, the performance of the proposed DVCC is suitable for many applications, e.g., implantable biomedical devices, where the processing signal possesses low amplitude and frequency in the ranges of millivolt and kilohertz, respectively [3-7].

The paper is organized as follows: to comprehend the DVCC principle and its non-ideal model, Sections 2 and 3 are presented. The QFG DVCC is employed in the design of a high-performance quadrature oscillator, which is given in Sections 4 and 5. At the end of the paper, the conclusion is provided.

\section{The proposed QFG-DVCC realization}

The Differential Voltage Current Conveyor (DVCC) is a powerful current-mode building block with the benefit of providing a multitude of interesting applications, e.g., in filters, oscillators, current-mode integrators, current-mode differentiators, instrumentation

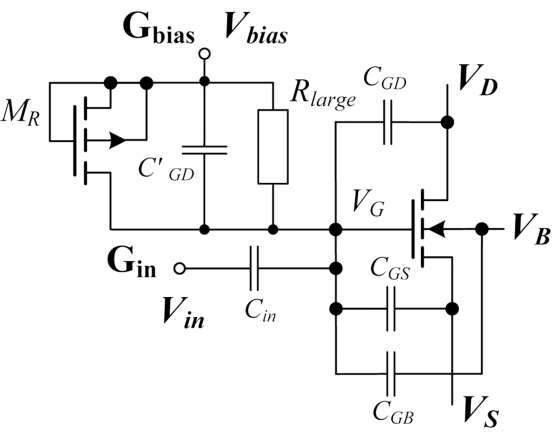

(c)

Figure 1. Single-input QFG MOST: (a) Symbolic with $R_{\mathrm{LARGE}}$, (b) symbolic with $M_{R}$, and (c) equivalent circuit of (b). 


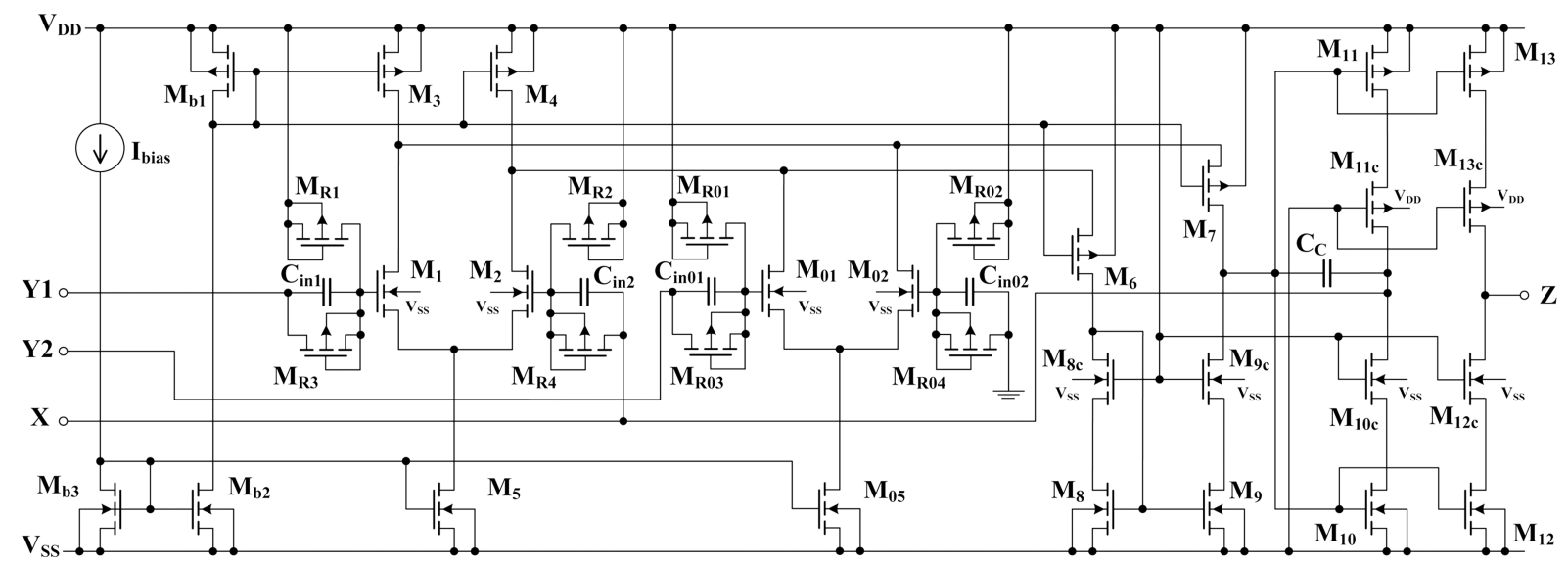

Figure 2. CMOS implementation of the novel LV LP DVCC based on QFG MOSTs.

amplifiers, [37-43]. The ideal DVCC is defined by the following hybrid matrix:

$$
\left(\begin{array}{c}
V_{X} \\
I_{Y 1} \\
I_{Y 2} \\
I_{Z}
\end{array}\right)=\left(\begin{array}{cccc}
0 & 1 & -1 & 0 \\
0 & 0 & 0 & 0 \\
0 & 0 & 0 & 0 \\
1 & 0 & 0 & 0
\end{array}\right)\left(\begin{array}{c}
V_{X} \\
V_{Y 1} \\
V_{Y 2} \\
V_{Z}
\end{array}\right)
$$

The novel DVCC based on QFG MOSTs is presented in Figure 2. The proposed structure is composed of input, second, and output stages. The input stage consists in differential pair transistors $M_{1}-M_{2}$ and $M_{01}-M_{02}$ whose gate terminals are connected to the positive supply voltage via reverse-biased diode connected transistors $M_{R 1}-M_{R 2}$ and $M_{R 01}-M_{R 02}$, respectively. This type of connection assures that the differential pair transistors are placed in the saturation region and the threshold voltage is shifted away from the signal path. Transistors $M_{b 1}, M_{b 2}, M_{b 3}, M_{3}, M_{4}$, $M_{5}$, and $M_{05}$ are used to set up the bias currents necessary for the differential stages. In addition, transistors $M_{6}$ and $M_{7}$ provide a level shift function, and the cascoded current mirrors $M_{8}, M_{9}, M_{8 c}$, and $M_{9 c}$ provide a differential to single-ended conversion. The second stage consists in transistors $M_{10}, M_{10 c}$, $M_{11}$, and $M_{11 c}$. The proposed circuit is enhanced by using cascode structures $M_{12}-M_{12 c}$ and $M_{13}-M_{13 c}$, rather than the simple one, to obtain an accurate copy of the input current and higher output impedance desirable for easy cascading; these transistors form the output stage of the proposed DVCC. The small Miller compensation network with $C_{C}$ of $0.01 \mathrm{pF}$ is employed for stability and for ensuring a good transient response with $10 \mathrm{pF}$ load capacitance. The optimal transistor aspect ratios are given in Table 1 .

It is observable in Figure 1(c) that the input of the QFG is connected in a high-pass filter configuration with cut-off frequency equal to:

$$
f_{c}=\frac{1}{2 \pi R_{\text {large }} C_{\text {total }}},
$$

Table 1. Component values and transistor aspect ratios from Figure 2.

\begin{tabular}{lc}
\hline \multicolumn{1}{c}{ QFG-DVCC } & $\begin{array}{c}\boldsymbol{W} / \boldsymbol{L} \\
{[\boldsymbol{\mu \mathbf { m } / \boldsymbol { \mu } \mathbf { m } ]}}\end{array}$ \\
\hline$M_{1}, M_{2}, M_{01}, M_{02}$ & $3 / 0.5$ \\
$M_{3}, M_{4}$ & $13 / 1$ \\
$M_{5}, M_{05}$ & $4 / 0.6$ \\
$M_{6}, M_{7}$ & $20 / 1$ \\
$M_{8}, M_{9}$ & $8 / 1$ \\
$M_{8 c}, M_{9 c}, M_{b 2}, M_{b 3}$ & $2 / 1$ \\
$M_{10}, M_{12}, M_{10 c}, M_{12 c}$ & $10 / 0.5$ \\
$M_{11}, M_{13}, M_{11 c}, M_{13 c}$ & $30 / 0.5$ \\
$M_{b 1}$ & $6 / 1$ \\
$M_{R 1}-M_{R 4}, M_{R 01}-M_{R 04}$ & $5 / 1$ \\
\hline$C_{c}=0.01 \mathrm{pF}, C_{i n 1}, C_{i n 2}, C_{i n 01}, C_{i n 02}=0.2 \mathrm{pF}$, \\
$I_{\text {bias }}=1 \mu \mathrm{A}$
\end{tabular}

where $C_{\text {total }}$ is the total capacitance (Eq. (2)). To attain low cut-off frequency in the milli- hertz range, one should use a substantially large resistance value and moderately small capacitance. As a consequence, the filter will not affect the circuit performance working in higher frequencies (higher than the $\mathrm{mHz}$ range) [36].

However, to keep proper behavior of the DVCC, i.e., to also process the dc voltage, reverse-biased diode connected transistors $M_{R 3}, M_{R 4}, M_{R 03}$, and $M_{R 04}$ are connected in parallel to the input capacitors $C_{i n 1}$, $C_{i n 2}, C_{i n 01}$, and $C_{i n 02}$, respectively. These connections create uncompensated voltage dividers. However, this effect, thanks to the internal feedback from the output of the second stage to $X$ terminal, is suppressed [44].

It is worth mentioning that for some DVCC applications, there is a need to have both polarities of output current $I_{Z}$ or multiple outputs $I_{Z+}$ and/or $I_{Z-}$. This could be achieved by simply using current mirrors technique to copy and/or invert polarity of the output current $I_{Z}$ without affecting the performance of $X, Y 1$, 
$Y 2$, and $Z$ terminals. This is the case for our proposed application example as a quadrature oscillator.

To finalize the verification of the proposed DVCC, all the required simulations are demonstrated in this part using 0.18- $\mu \mathrm{m}$ TSMC CMOS technology with the spice model shown in Figure 3.

Figure 4 depicts the frequency dependence of parasitic impedance for $X$ and $Z$ terminals. The parasitic impedance for $X$ and $Z$ terminals is apparently low and high, respectively. More specifically, the impedance value at low frequency is $31 \mathrm{~dB}$ at $X$ input terminal, and the value of the output impedance at $Z$ terminal is $135 \mathrm{~dB}$; consequently, the high output impedance of $Z$ terminal offers a solution to overcome the loading effect issue without the need for an additional buffer.

Figure 5 clarifies the frequency response of current gain $I_{Z} / I_{X}$ and voltage gains $V_{X} / V_{Y 1}, V_{X} / V_{Y 2}$. The DVCC provides $19 \mathrm{MHz}$ bandwidth at $-3 \mathrm{~dB}$. Figure 6 depicts the DC curves of $I_{X}$ versus $I_{Z}$ and related current errors $\Delta I=I_{Z}-I_{X}$ for various temperatures of 0,27 , and $60^{\circ} \mathrm{C}$; it is evident that for the input current range of -50 to $50 \mu \mathrm{A}$, the current errors are negligible.

Figure 7 illustrates the DC curve of $V_{X}$ versus $V_{Y 1}$ assuming that $Y 2$ terminal is grounded. This curve shows an attractive rail-to-rail operating range. The low values of voltage errors $\boldsymbol{\Delta} \boldsymbol{V}=V_{Y 1}-V_{X}$ for temperatures of 0,27 , and $60^{\circ} \mathrm{C}$ are illustrated as well.

As shown in Figure 8, the voltage at $X$ terminal follows the voltage difference of terminals $Y 1$ and $Y 2$ in a wide range; while stepping $V_{Y 2}$ to various values of $[-150,-100,-50,0,50,100,150] \mathrm{mV}$.

Table 2 sums up the key design characteristics of the proposed QFG-DVCC circuit and the comparison with some other recently published works. It is notable that the proposed QFG-DVCC can be boastful of its attractive performances, especially the low supply voltage of $\pm 400 \mathrm{mV}$ and the ultra-low-power consumption of $6.6 \mu \mathrm{W}$, which are the primary aim of this design.

\section{Non-ideal analysis of DVCC}

Transistors mismatch, channel length modulation effect, and parasitic capacitances lead to difference between the ideal DVCC and the real one; the parasitic impedance of DVCC and the non-ideal gain effect adversely affect the circuit behavior and may pose deviations in the circuit characteristics. However, these effects could be minimized and unnoticeable when proper circuit design, layout design rules, and connection of the external passive elements with DVCC are applied. One of the suitable connections to absorb parasitic capacitances is that of grounded capacitors at $Y$ and $Z$ terminals, as a result of which a parallel connection of the low value of the parasitic capacitance with the external grounded capacitor is obtained. In the same way, the parasitic resistances at $X$ termi-

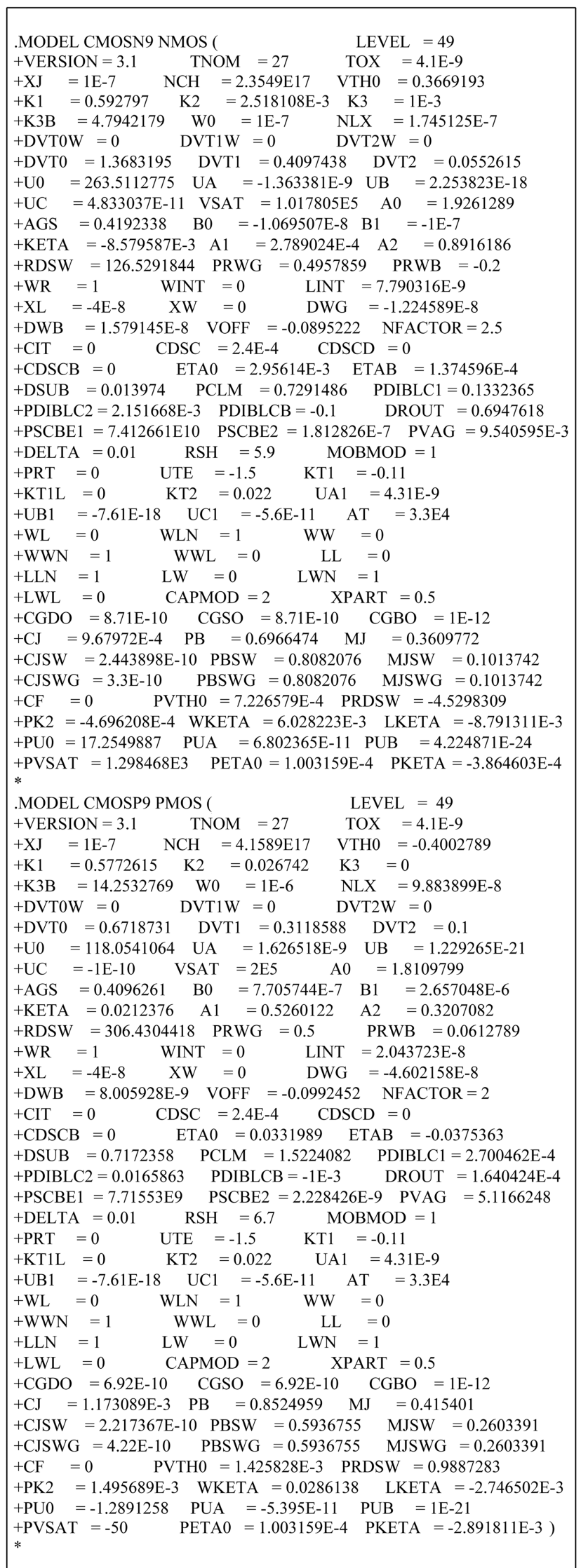

Figure 3. Mosis parametric test results. Vendor: TSMC; feature size: 0.18 microns. 
Table 2. Simulation results of the QFG-DVCC and comparison with other LV LP DVCCs in the literature.

\begin{tabular}{|c|c|c|c|c|c|}
\hline Characteristics & Proposed & {$[21]$} & {$[40]$} & {$[41]$} & {$[42]$} \\
\hline Supply voltage & $\pm 0.4 \mathrm{~V}$ & $\pm 0.5 \mathrm{~V}$ & $\pm 1.5 \mathrm{~V}$ & $\pm 1.5 \mathrm{~V}$ & $\pm 0.9 \mathrm{~V}$ \\
\hline Power consumption & $6.6 \mu \mathrm{W}$ & $10 \mu \mathrm{W}$ & $1.74 \mathrm{~mW}$ & $2 \mathrm{~mW}$ & $0.462 \mathrm{~mW}$ \\
\hline DC current range & -50 to $+50 \mu \mathrm{A}$ & -30 to $+30 \mu \mathrm{A}$ & -1 to $1 \mathrm{~mA}$ & - & \\
\hline $\mathrm{DC}$ voltage range & -400 to $+400 \mathrm{mV}$ & -500 to $+500 \mathrm{mV}$ & -900 to $900 \mathrm{mV}$ & - & - \\
\hline $3 \mathrm{~dB}$ bandwidth $I_{Z} / I_{X}$ & $19 \mathrm{MHz}$ & $10.2 \mathrm{MHz}$ & $120 \mathrm{MHz}$ & $80 \mathrm{MHz}$ & $598.4 \mathrm{MHz}$ \\
\hline Current gain $I_{Z} / I_{X}$ & 1 & 1 & - & - & 0.9999 \\
\hline $3 \mathrm{~dB}$ bandwidth $V_{X} / V_{Y 1}, V_{X} / V_{Y 2}$ & $19 \mathrm{MHz}$ & $2.9 \mathrm{MHz}, 3.3 \mathrm{MHz}$ & $85 \mathrm{MHz}$ & - & $\begin{array}{l}588.84 \mathrm{MHz} \\
605.86 \mathrm{MHz}\end{array}$ \\
\hline Voltage gain $V_{X} / V_{Y 1}, V_{X} / V_{Y 2}$ & 1 & 1 & - & - & $1,0.999$ \\
\hline Node $X$ parasitic resistance: $R_{X} / L_{X}$ & $40 \Omega / 0.5 \mathrm{mH}$ & $70 \Omega / 3 \mathrm{mH}$ & $9 \Omega /-$ & $0.6 \mathrm{k} \Omega /-$ & $150.2 \Omega / 4.16 \mu \mathrm{H}$ \\
\hline $\begin{array}{l}\text { Nodes } Y 1, Y 2 \text { parasitic resistances: } \\
R_{Y 1} / C_{Y 1}, R_{Y 2} / C_{Y 2}\end{array}$ & $2.3 \mathrm{G} \Omega / 70 \mathrm{fF}$ & $\begin{array}{l}0.933 \mathrm{~T} \Omega / 0.17 \mathrm{pF} \\
0.622 \mathrm{~T} \Omega / 0.25 \mathrm{pF}\end{array}$ & - & - & $\begin{array}{l}29.08 \mathrm{~T} \Omega / 3.86 \mathrm{fF}, \\
29.08 \mathrm{~T} \Omega / 3.86 \mathrm{fF}\end{array}$ \\
\hline Node $Z$ parasitic resistance: $R_{Z} / C_{Z}$ & $6.26 \mathrm{M} \Omega / 50 \mathrm{fF}$ & $55.7 \mathrm{M} \Omega / 7.7 \mathrm{fF}$ & - & $14 \mathrm{k} \Omega /-$ & $5.03 \mathrm{M} \Omega / 0.024 \mathrm{pF}$ \\
\hline CMOS technology & $0.18 \mu \mathrm{m}$ & $0.18 \mu \mathrm{m}$ & $0.25 \mu \mathrm{m}$ & $0.25 \mu \mathrm{m}$ & $0.18 \mu \mathrm{m}$ \\
\hline Obtained results & Simulated & Simulated & Simulated & Simulated & Simulated \\
\hline
\end{tabular}

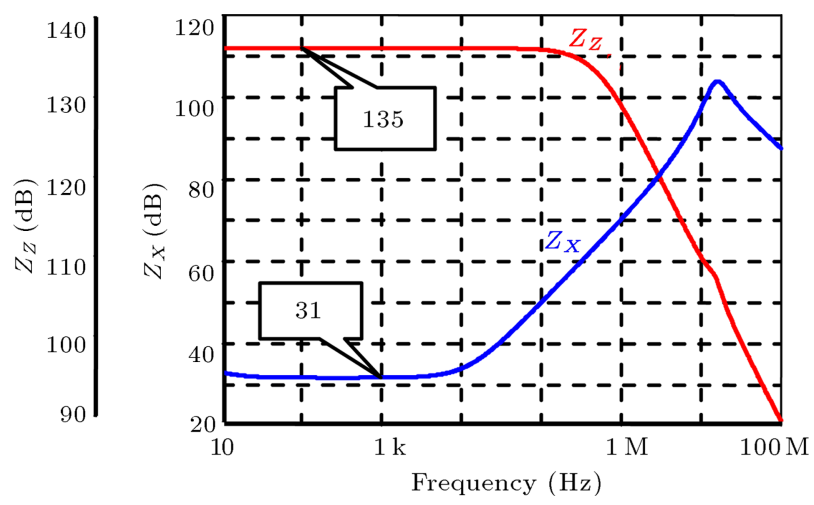

Figure 4. Frequency dependence of parasitic impedance of $X$ and $Z$ terminals.

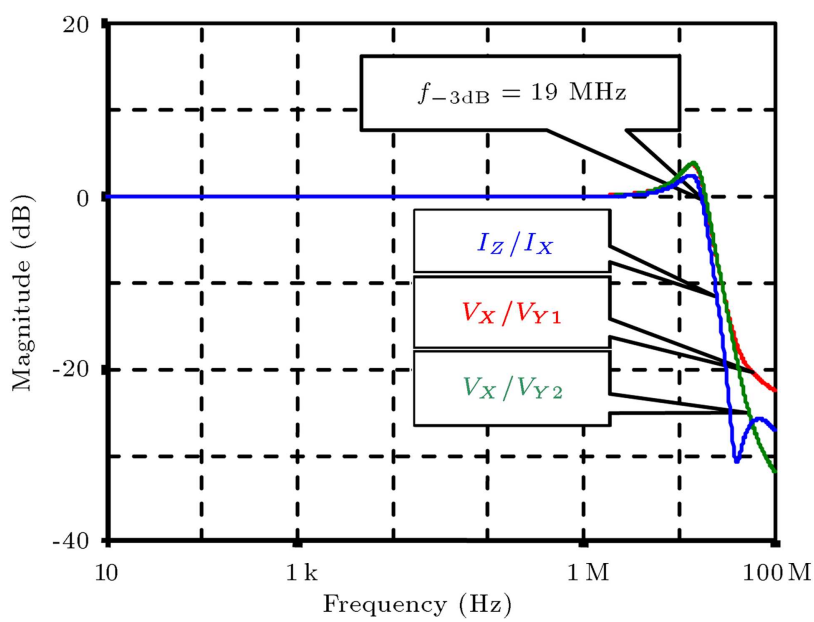

Figure 5. Frequency response of current gain $I_{Z} / I_{X}$ and voltage gains $V_{X} / V_{Y 1}, V_{X} / V_{Y 2}$.

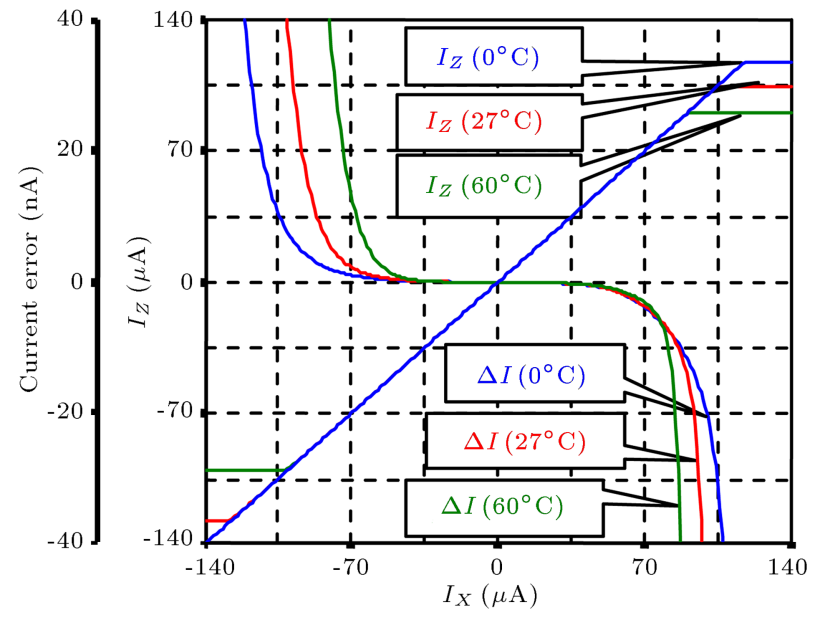

Figure 6. DC curves $I_{Z}$ versus $I_{X}$ and the current error for temperatures of 0,27 , and $60^{\circ} \mathrm{C}$.

nal can be absorbed by a serial connection of this small parasitic resistance with the external grounded one [42].

Owing to these reasons, the block diagram of DVCC and its non-ideal model are studied and shown in Figure 9. The non-ideal model of DVCC consists of an ideal DVCC in addition to current (CF), Voltage Follower (VF), and the parasitic impedances of DVCC terminals. The parasitic impedances to each of the $Y 1, Y 2$, and $Z$ terminals obtain a parallel combination of parasitic resistance and capacitance, whereas at $X$ terminal, a serial combination of parasitic resistance and inductance in parallel to parasitic capacitance appears; the value of this parasitic capacitance is 


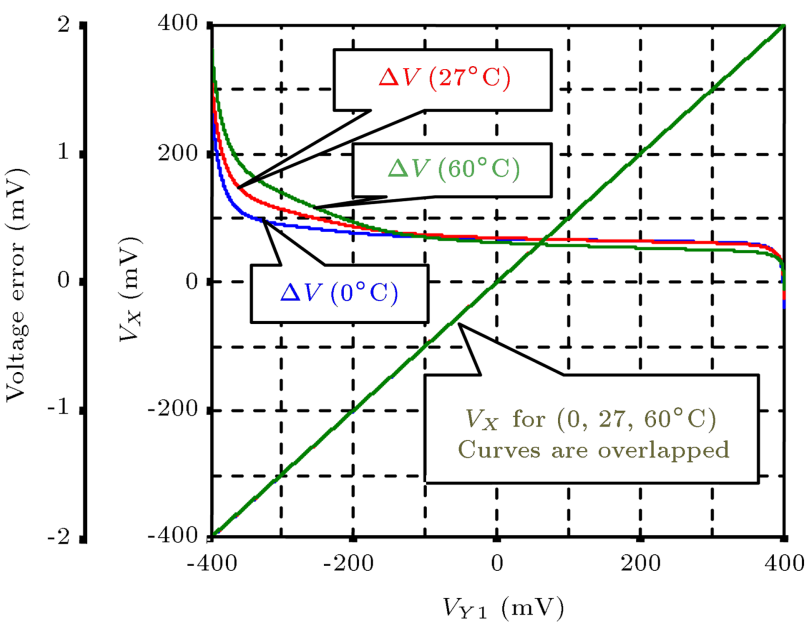

Figure 7. DC curves $V_{X}$ versus $V_{Y 1}$ (with $V_{Y 2}$ grounded), showing the rail-to-rail operating range and the voltage error for temperatures of 0,27 , and $60^{\circ} \mathrm{C}$.

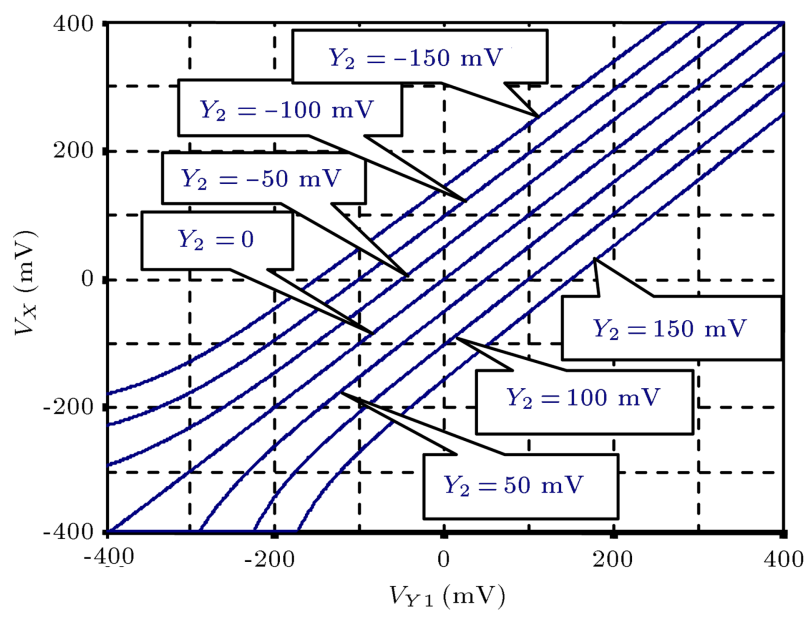

Figure 8. $7 \mathrm{DC}$ curves $V_{X}$ versus $V_{Y 1}$ for $V_{Y 2}=[-150$, $-100,-50,0,50,100,150] \mathrm{mV}$. usually omitted as it affects response at a very high frequency. However, the values of these parasitic components could be easily calculated as in [21].

Ideally, the $X$ terminal exhibits zero resistance, while the $Y$ and $Z$ terminals exhibit infinite resistance; practically, these resistances assume the finite value depending upon the internally used device parameters of the related active element $[21,37]$. However, the real QFG-DVCC can be characterized by the following hybrid matrix:

$$
\left(\begin{array}{c}
V_{X} \\
I_{Y 1} \\
I_{Y 2} \\
I_{Z}
\end{array}\right)=\left(\begin{array}{cccc}
Z_{X} & \beta_{1}(s) & -\beta_{2}(s) & 0 \\
0 & 1 / Z_{Y 1} & 0 & 0 \\
0 & 0 & 1 / Z_{Y 2} & 0 \\
\alpha(s) & 0 & 0 & 1 / Z_{z}
\end{array}\right)\left(\begin{array}{c}
I_{X} \\
V_{Y 1} \\
V_{Y 2} \\
V_{Z}
\end{array}\right)
$$

The voltage and current transfers of the DVCC are given by $\beta_{1}(\mathrm{~s}), \beta_{2}(\mathrm{~s})$, and $\alpha(\mathrm{s})$, respectively:

$$
\begin{aligned}
& \beta_{1}(s)=\frac{\beta_{01}}{1+s / w_{\beta 1}}, \\
& \beta_{2}(s)=\frac{\beta_{02}}{1+s / w_{\beta 2}}, \\
& \alpha(s)=\frac{\alpha_{0}}{1+s / w_{\alpha}},
\end{aligned}
$$

where $\beta_{01}, \beta_{02}$, and $\alpha_{0}$ are the values of these transfers at low frequencies and $w_{\beta 1}, w_{\beta 2}$, and $w_{\alpha}$ represent their corresponding pole frequencies. The low-frequency voltage and current transfers are assumed to be $\beta_{0 j}=$ $1-\varepsilon v j$ for $j=1,2$ and $\alpha_{0}=1-\varepsilon i$, where $\varepsilon v j$ and $\varepsilon i$ $(|\varepsilon v j|<<1$ and $|\varepsilon i|<<1)$ represent voltage and current tracking errors of the QFG-DVCC, respectively. For a small-signal equivalent circuit, a straightforward analysis brings the following expressions to $\beta_{01}, \beta_{02}$, and $\alpha_{0}$.

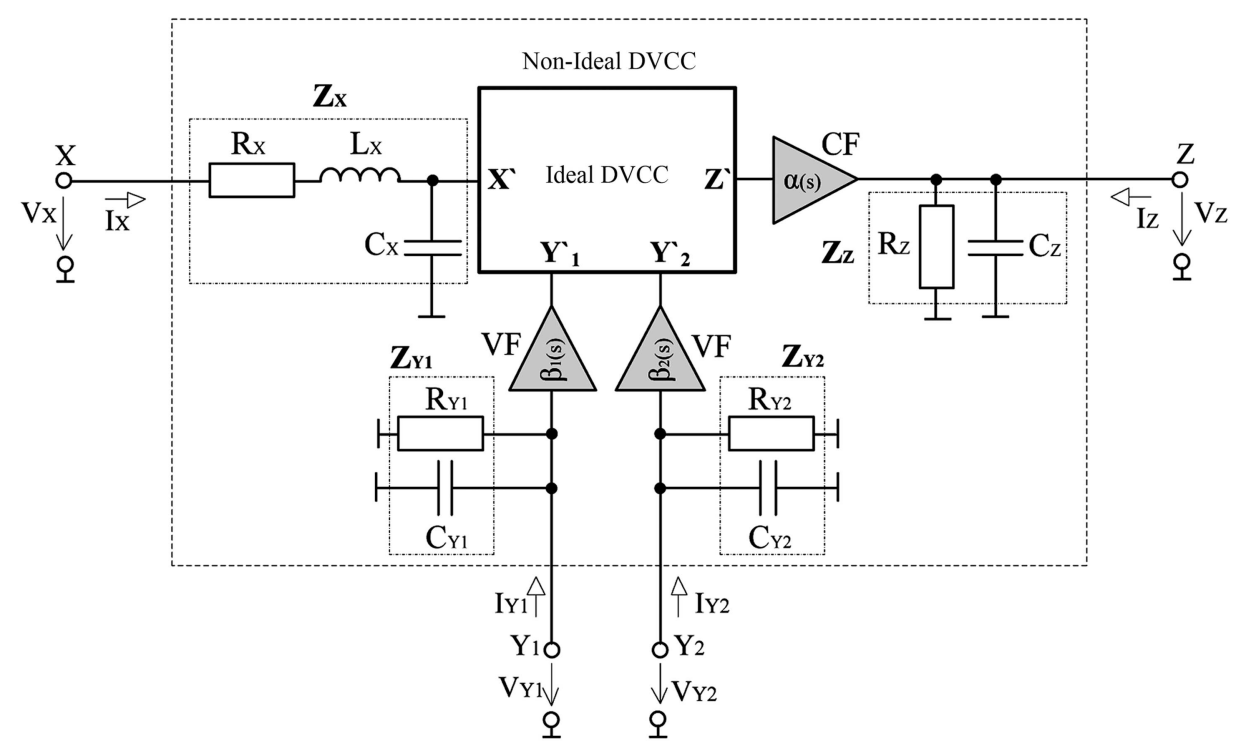

Figure 9. Model of non-ideal DVCC. 
The voltage transfers $\beta_{01}$ and $\beta_{02}$ are:

$$
\begin{aligned}
\beta_{01}= & \frac{V_{X}}{V_{Y 1}} \\
= & \frac{g_{m, Q F G, M 1} r_{\text {out } 1}\left(g_{m M 10}+g_{m M 11}\right) r_{\text {out } 2}}{1+\left(g_{m, Q F G, M 2} r_{\text {out } 1}\left(g_{m M 10}+g_{m M 11}\right) r_{\text {out } 2}\right)} \\
\approx & \frac{g_{m, Q F G, M 1}}{g_{m, Q F G, M 2}} \approx 1, \\
\beta_{02}= & \frac{V_{X}}{V_{Y 2}} \\
& =\frac{g_{m, Q F G, M 01} r_{\text {out } 1}\left(g_{m M 10}+g_{m M 11}\right) r_{\text {out } 2}}{1+\left(g_{m, Q F G, M 2} r_{\text {out } 1}\left(g_{m M 10}+g_{m M 11}\right) r_{\text {out } 2}\right)} \\
& \approx \frac{g_{m, Q F G, M 01}}{g_{m, Q F G, M 2}} \approx 1,
\end{aligned}
$$

where $r_{\text {out } 1}$ and $r_{\text {out } 2}$ are the output impedances of the first and second stages of QFG-DVCC and given as:

$$
\begin{aligned}
& r_{\text {out } 1} \\
& =\frac{1}{\frac{g_{o, M 9 c} g_{o, M 9}}{g_{m, M 9 c}}+\frac{g_{o, M 7}\left(g_{o, M 3}+g_{o, Q F G, M 1}+g_{o, Q F G, M 02}\right)}{g_{m, M 7}+g_{m b, M 7}}}, \\
& r_{\text {out } 2}=\frac{1}{\frac{g_{o, M 10 c} g_{o, M 10}}{g_{m, M 10 c}}+\frac{g_{o, M 11 c} g_{o, M 11}}{g_{m, M 11 c}+g_{m b, M 11}}} .
\end{aligned}
$$

Then, the current transfer $\alpha_{0}$ is:

$$
\alpha_{0}=\frac{I_{Z}}{I_{X}}=\frac{g_{m, M_{12}}+g_{m, M_{13}}}{g_{m, M_{10}}+g_{m, M_{11}}} \approx 1 .
$$

The impedance of the $Y 1$ and $Y 2$ terminals of the proposed QFG-DVCC is very high, because the quasifloating-gate of the MOS transistor is used as the input to the amplifier stage. The resistance of the remaining terminal $X$ is as follows:

$$
R_{X}=\frac{1}{g_{m, Q F G, M 1} r_{\text {out } 1}\left(g_{m, M 10}+g_{m, M 11}\right)} \text {. }
$$

Finally, the low-frequency resistance of the $Z$ terminal can be expressed as:

$$
R_{Z}=\frac{1}{\frac{g_{o, M 12 c} g_{o, M 12}}{g_{m, M 12 c}}+\frac{g_{o, M 13 c} g_{o, M 13}}{g_{m, M 13 c}+g_{m b, M 13 c}}} .
$$

In Eqs. (9)-(15), $g_{m}$ and $g_{m b}$ denote the gate and bulk transconductances of MOST, $g_{m, Q F G}$ denotes the transconductance of QFG-MOST, and $g_{0}$ is the output conductance of the MOST.

\section{Quadrature oscillator as an example of application}

As an application example of the novel QFG DVCC with low voltage and ultra-low power, a quadrature

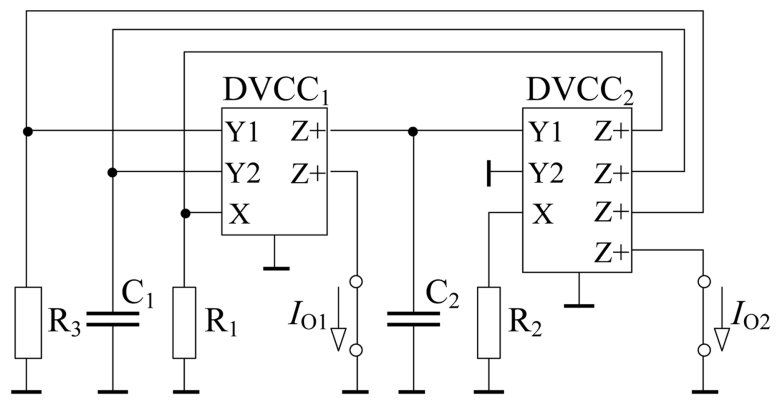

Figure 10. The proposed oscillator using two DVCCs.

oscillator using two active elements has been designed. Note that one of the first current-mode quadrature oscillators based on DVCC was presented in [45]. Quadrature sinusoidal oscillators are important circuits for various communication applications, wherein there is a requirement for multiple sinusoids that are $90^{\circ}$ phase shifted, e.g., in quadrature mixers and singlesideband modulators or for measurement purposes in the vector generator or selective voltmeters [46,47]. Therefore, quadrature oscillators are widely used in many communications, signal processing, and instrumentation systems $[48,49]$.

The proposed structure is shown in Figure 10 and it can be seen that all passive elements are grounded, which make the circuit interesting for integrated implementation.

A routine analysis of the oscillator yields the following Characteristic Equation (CE):

$$
C E: s^{2} C_{1} C_{2} R_{1} R_{2}+s C_{1}\left(R_{1}-R_{3}\right)+1=0 .
$$

From Eq. (16), the Condition of Oscillation (CO) and the Frequency of Oscillation (FO) can be evaluated as:

$$
\begin{aligned}
& C O: R_{1} \leq R_{3}, \\
& F O: f_{0}=\frac{1}{2 \pi} \sqrt{\frac{1}{C_{1} C_{2} R_{1} R_{2}}} .
\end{aligned}
$$

From Relations (17) and (18), it is evident that the condition of oscillation can be controlled by $R_{3}$ without affecting the oscillation frequency. Similarly, by varying the value of the resistor $R_{2}$, only the oscillation frequency is affected.

The relationship between the output currents can be given as:

$$
I_{\mathrm{O} 1}=j k I_{\mathrm{O} 2},
$$

where:

$$
k=\omega_{0} C_{2} R_{2},
$$

ensuring that output currents $I_{O 1}$ and $I_{O 2}$ are quadrature (the phase difference $\phi=90^{\circ}$ ) and have equal amplitudes for $k=1$. 


\section{Non-ideal analysis and sensitivity study}

In this section, the non-idealities of the QFG DVCC shown in Eq. (5) and their influences on the proposed quadrature oscillator are studied. Assuming the nonideal behavior of the active elements (non-unity voltage and current gains) and excluding parasitic resistances and capacitances, the characteristic equation, condition of oscillation, and frequency of oscillation change to:

$$
\begin{array}{ll}
C E: \quad & s^{2} C_{1} C_{2} R_{1} R_{2}+\alpha_{01} \alpha_{02} \beta_{021} s C_{1}\left(R_{1}-\beta_{011} R_{3}\right) \\
& +\alpha_{01} \alpha_{02} \beta_{012} \beta_{021}=0, \\
C O: & R_{1} \leq \beta_{011} R_{3}, \\
F O: & f_{0}=\frac{1}{2 \pi} \sqrt{\frac{\alpha_{01} \alpha_{02} \beta_{012} \beta_{021}}{C_{1} C_{2} R_{1} R_{2}}}
\end{array}
$$

and the affected relation between the output currents is:

$$
I_{\mathrm{O} 1}=j k^{\prime} I_{\mathrm{O} 2}, \text { where } \quad k^{\prime}=\frac{1}{\alpha_{02} \beta_{021}} \omega_{0} C_{2} R_{2},
$$

which means that the oscillator is still quadrature.

In Relations (21)-(23), it can be seen that the non-ideal behavior of the active elements affects both the condition and the frequency of oscillation; however, since the value of the resistor $R_{3}$ is always significantly higher than that of the resistor $R_{1}$, there is no problem in fulfilling the CO.

A sensitivity study forms an important index of the performance of any active network. The formal definition of sensitivity is as follows:

$$
S_{x}^{F}=\frac{x}{F} \frac{\partial F}{\partial x},
$$

where $F$ and $x$ represent the frequency of oscillation $f_{0}$ and any of the passive elements $\left(C_{1}, C_{2}, R_{1}, R_{2}\right)$ or active parameters $\alpha_{0 i j}, \beta_{0 i j}$ ). Using the above definition, the active and passive sensitivities of the proposed circuit are given as:

$$
\left|S_{\alpha_{01}, \alpha_{02}, \beta_{012}, \beta_{021}, C_{1}, C_{2}, R_{1}, R_{2}}^{f_{0}}\right|=0.5 \text {. }
$$

Eq. (26) indicates that all the $f_{0}$ passive and active sensitivities are 0.5 in absolute value; hence, the circuit exhibits an attractive sensitivity performance.

For a complete analysis of the circuit, it is also important to take the main parasitic impedances and capacitances of the QFG DVCC into account. The proposed oscillator with the parasitic elements is shown in Figure 11. An analysis shows the dominant effect of the following parasitics:

- The non-zero input parasitic resistances $R_{x 1}$ and

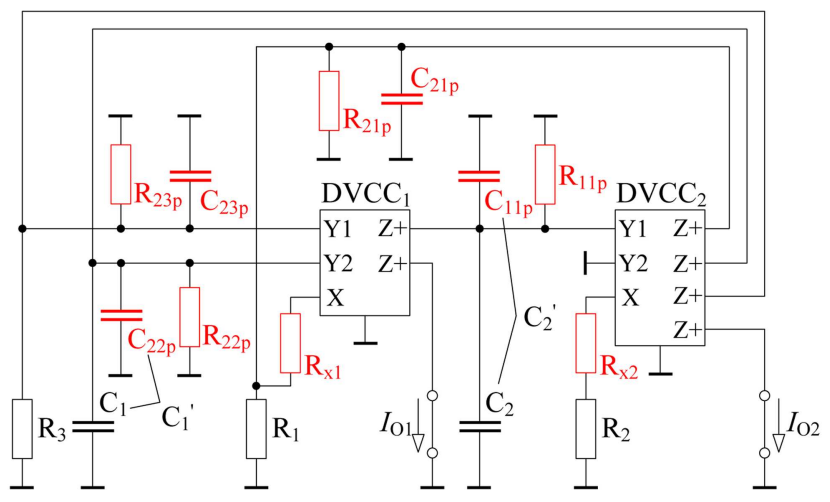

Figure 11. The proposed oscillator including the parasitic elements of the DVCC.

$R_{x 2}$ appearing at $X$ terminals of DVCCs, where $R_{x 2}$ can be absorbed in the external resistor $R_{2}$, which is in serial with it;

- The parasitic resistance $R_{i j p}$ appearing between the high output impedance terminal $Z_{j}$ (where $j=1,2$, $3,4)$ of the $i$ th DVCC and ground. Excluding $R_{21 p}$, parasitic resistances are in parallel combination with those appearing at high input impedance terminal $Y_{i k}$ (where $k=1,2$ ), which are as follows:

$$
\begin{gathered}
R_{11 p}=R_{y 21}\left\|R_{z 11}, R_{22 p}=R_{y 12}\right\| R_{z 22} \text { and } \\
R_{23 p}=R_{y 11} \| R_{z 23} .
\end{gathered}
$$

Similarly, the parasitic capacitance $C_{i j p}$ appears between the high output impedance terminal $Z_{j}$ and ground. The parasitic capacitances $C_{22 p}$ and $C_{11 p}$ are absorbed into external capacitors $C_{1}$ and $C_{2}$, respectively, as they appear in parallel with them, where $C_{22 p}=C_{y 12}+C_{z 22}$ and $C_{11 p}=C_{y 21}+$ $C_{z 11}$. In addition, the parasitic capacitances at high impedance terminals $C_{y 11}$ and $C_{z 23}$ are in shunt labeled as $C_{23 p}$ in Figure 12 .

The effects of these parasitics can be alleviated

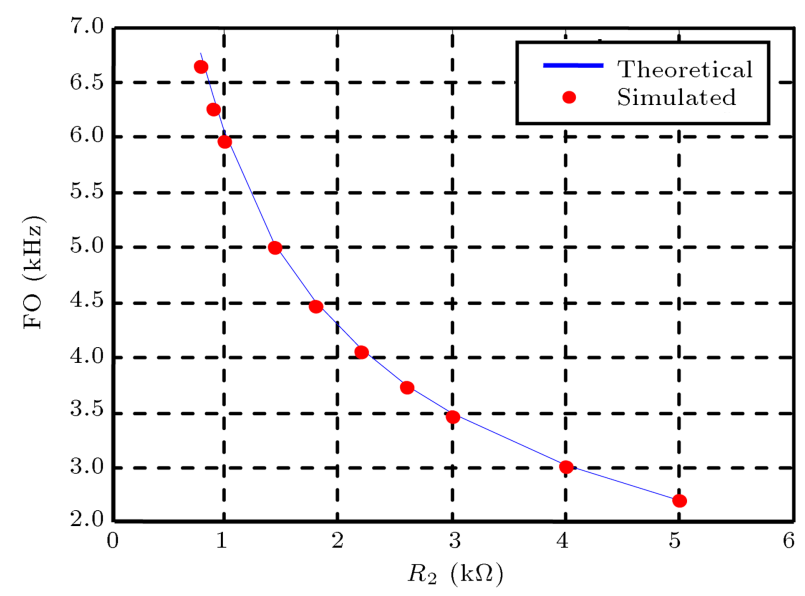

Figure 12. The variation of FO by $R_{2}$. 
by considering the external capacitors $C_{1}$ and $C_{2}>>$ $C_{i j p}$; external resistors $R_{1}, R_{3}<<R_{21 p}$, and $R_{23 p}$; and the operating frequency $\omega_{0}>\max \left[1 / R_{22 p}\left(C_{1}+C_{22 p}\right)\right.$, $\left.1 / R_{11 p}\left(C_{2}+C_{11 p}\right)\right]$.

Considering the aforementioned parasitic effects, excluding parasitic resistances, and assuming that the current and voltage gains of active elements used are unities $\alpha_{0 i j}=1, \beta_{0 i 1}=1$, and $\beta_{0 i 2}=-1$ ), the ideal characteristic equation in Eq. (16) turns into:

$$
\begin{aligned}
C E: & s^{3} C_{1}{ }^{\prime} C_{2}{ }^{\prime} C_{3 p} R_{1} R_{2} R_{3}+s^{2} R_{1} \\
& {\left[C_{1}{ }^{\prime}\left(C_{2}{ }^{\prime} R_{2}+C_{3 p} R_{3}-C_{4 p} R_{3}\right)+C_{3 p} C_{4 p} R_{3}\right] } \\
& +s\left[C_{1}{ }^{\prime}\left(R_{1}-R_{3}\right)+C_{3 p} R_{3}+C_{4 p} R_{1}\right]+1=0,
\end{aligned}
$$

and the CO and FO in Relations (17) and (18) are modified as:

$$
\begin{aligned}
C O: & C_{1}{ }^{\prime 2} C_{2}{ }^{\prime} R_{1} R_{2}+C_{1}{ }^{2}\left(C_{3 p} R_{1} R_{3}+C_{4 p} R_{3}{ }^{2}\right) \\
& +C_{1}{ }^{\prime} C_{2}{ }^{\prime} C_{4 p} R_{1} R_{2}+C_{1}{ }^{\prime}\left[2 C_{3 p} C_{4 p} R_{1} R_{3}+C_{3 p}{ }^{2} R_{3}{ }^{2}\right] \\
& +C_{3 p} C_{4 p} R_{3}\left(C_{3 p} R_{3}+C_{4 p} R_{1}\right) \leq C_{1}{ }^{2} C_{2}{ }^{\prime} R_{2} R_{3} \\
& +C_{1}{ }^{2} R_{3}\left(C_{4 p} R_{1}+C_{3 p} R_{3}\right) \\
& +R_{3} C_{1}{ }^{\prime}\left(2 C_{3 p} C_{4 p} R_{3}+C_{4 p}{ }^{2} R_{1}\right)
\end{aligned}
$$

$F O: f_{0}=\frac{1}{2 \pi}$

$$
\sqrt{\frac{1}{R_{1}} \cdot\left[\frac{1}{C_{1}{ }^{\prime} C_{2}{ }^{\prime} R_{2}+R_{3}\left[C_{1}^{\prime}\left(C_{3 p}-C_{4 p}\right)+C_{3 p} C_{4 p}\right]}\right]},
$$

where $C_{1}^{\prime}=C_{1}+C_{22 p}$ and $C_{2}^{\prime}=C_{2}+C_{11 p}$.

It is found that the parasitics affect both the condition and the frequency of oscillation. However, these deviations are very small and can be ignored, since the value of external capacitors is much greater than $C_{i j p}$, the value of external resistors used is much lower than $R_{i j p}$, and the proposed oscillator operating frequency is much lower than the operating frequency of parasitics.

\section{Simulation}

In order to verify the workability of the oscillator designed, it has been simulated using SPICE. To the CMOS structure of the current conveyor in Figure 2, additional $Z$-terminals are added to create the required feedbacks in the oscillator circuit as shown in Figure 10. The values of the passive elements are set as follows:

$$
C_{1}=C_{2}=22 n F, \quad R_{1}=1.43 \mathrm{k} \Omega, \quad R_{3}=2.00 \mathrm{k} \Omega .
$$

The resistor $R_{2}$ is used to control the frequency of

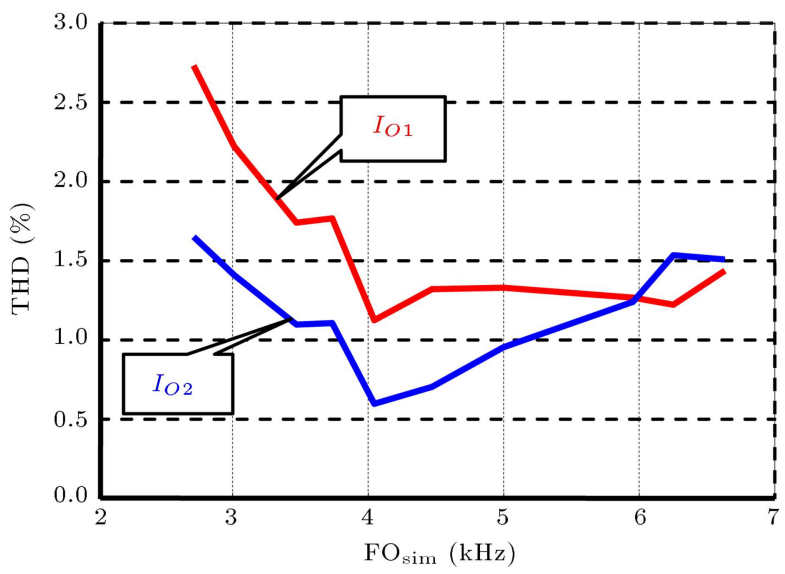

Figure 13. The variation of THD with the generated frequency.

oscillation and in simulations, it varies in the range of $0.8 \mathrm{k} \Omega$ to $5 \mathrm{k} \Omega$.

Comparison of the theoretical value of the generated frequency with those given by simulations is shown in Figure 12.

The variation of the total harmonic distortion of the output currents $I_{O 1}$ and $I_{O 2}$ is shown in Figure 13. If the generated frequency is low, the required value of resistor $R_{2}$ needs to be high (see Figure 13) and it comes to voltage saturations of the current conveyor DVCC2. For high frequencies, the value of the resistor $R_{2}$ needs to be low (see Figure 13) and it comes to current limitations of the current conveyor $\mathrm{DVCC}_{2}$. In both cases, the value of THD rises (Figure 13).

To complete the simulations, for $R_{2}=1.43 \mathrm{k} \Omega$ (i.e., $f_{\text {teor }}=5.06 \mathrm{kHz}$ ), the steady state of the generated currents is given in Figure 14. The corresponding spectrum of these signals is shown in Figure 15. The real value of the generated signal is $5 \mathrm{kHz}$ with THD of $1.33 \%$ and $0.96 \%$ for the currents $I_{O 1}$ and $I_{O 2}$, respectively. The Monte Carlo mismatch analysis (50 runs) of the corresponding spectra of the oscillator

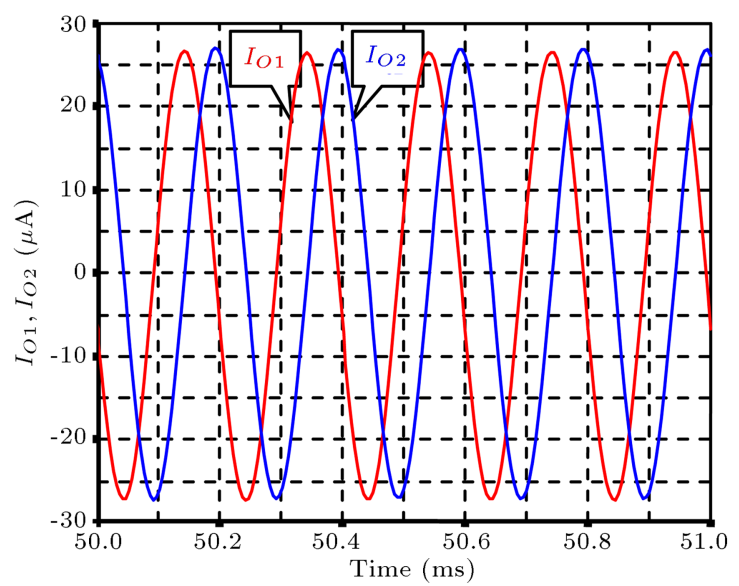

Figure 14. Steady-state oscillation waveforms of $I_{O 1}$ and $I_{O 2}$ for $R_{2}=1.43 \mathrm{k} \Omega$. 


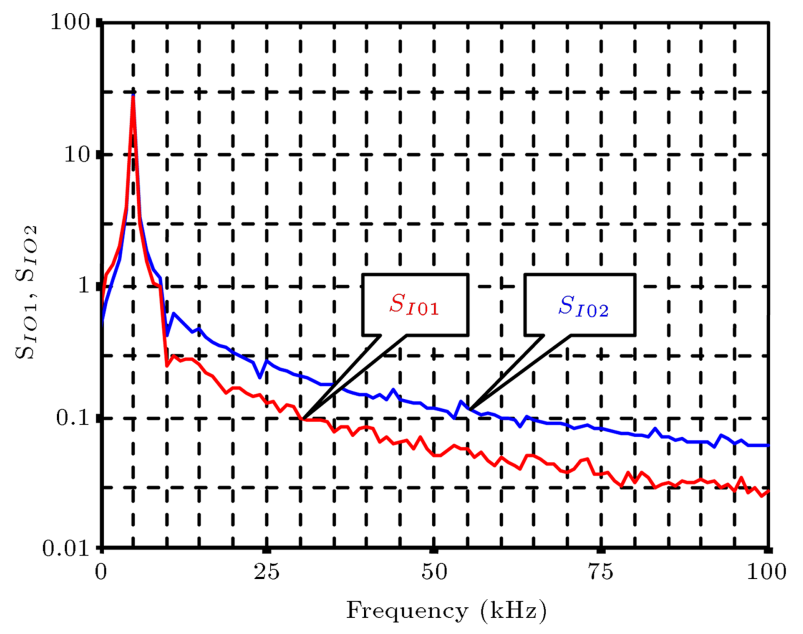

Figure 15. The corresponding spectra of the oscillator output currents $I_{O 1}$ and $I_{O 2}$.

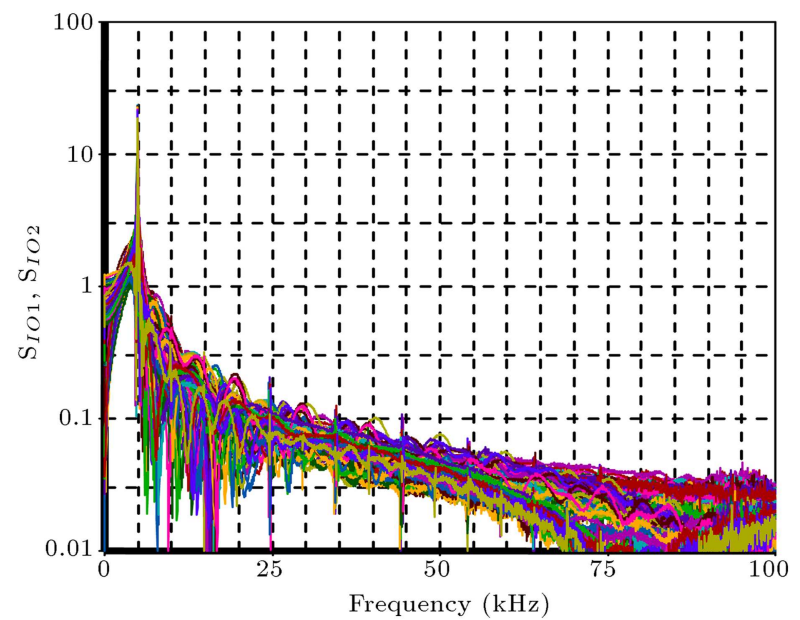

Figure 16. The Monte Carlo analysis of the corresponding spectra of the oscillator output currents $I_{O 1}$ and $I_{O 2}$.

output currents $I_{O 1}$ and $I_{O 2}$ is shown in Figure 16 and it confirms the low circuit sensitivity to transistor mismatches.

The quadrature relationship between the generated waveforms has been verified using Lissagous figure and shown in Figure 17. The total power dissipation of the oscillator is $0.28 \mathrm{~mW}$.

\section{Conclusion}

This paper deals with DVCC active element based on QFG technique. The proposed DVCC circuit can be used in various applications with attractive features, e.g., low-input impedance and high-output impedance, and it leads to superior properties in terms of cascading over other counterparts. Besides, lowvoltage and ultra-low-power properties were achieved with sustaining optimum circuit performance. At the end, the richness of the oscillator possibilities inherent

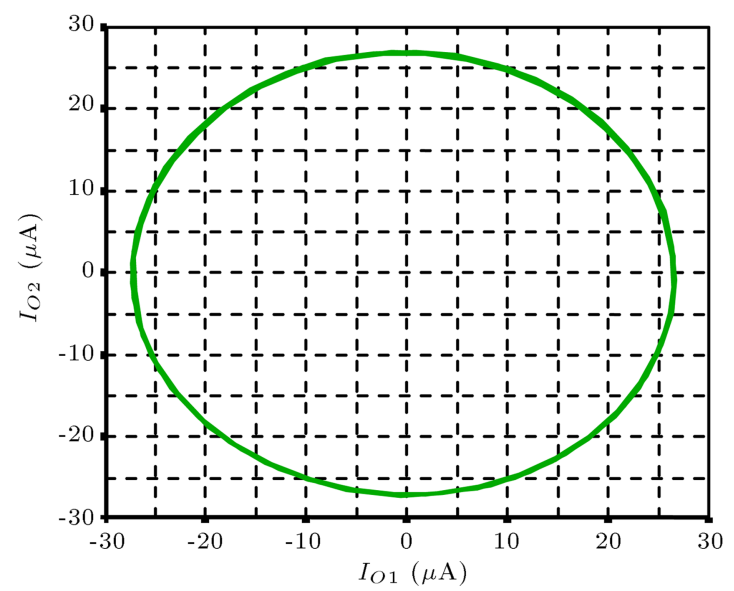

Figure 17. The quadrature relationship between the generated waveforms.

in producing quadrature signals and working with lowpower consumption was demonstrated as well.

\section{Acknowledgements}

The research was financed by the National Sustainability Program, under grant LO1401. For the research, infrastructure of the SIX Center was used.

\section{References}

1. Lasane, K. Integrated Analogue CMOS Circuits and Structures for Heart Rate Detectors and Other LowVoltage, Low-Power Applications, University of Oulu, pp. 1-118, Finland (2011).

2. Rajput, S.S. and Jamuar, S.S. "Low voltage analog circuit design techniques", IEEE Circuits and Systems Magazine, 2(1), pp. 24-42 (2002).

3. Hsu, C., Ho, M., Wu, Y., and Chen, T. "Design of low-frequency low-pass filters for biomedical applications", IEEE Asia Pacific Conference on Circuits and Systems, pp. 690-695 (2006).

4. Rodriguez-Villegasa, E., Corbishley, P., Martinez, C.L., and Rodriguez, T.S. "An ultra-low-power precision rectifier for biomedical sensors interfacing", Sensors and Actuators A: Physical, 153(2), pp. 222-229 (2009).

5. Veeravalli, A., Sanchez-Sinencio, E., and SilvaMartinez, J. "A CMOS transconductance amplifier architecture with wide tuning range for very low frequency applications", IEEE Journal of Solid-State Circuits, 37(6), pp. 776-781 (2002).

6. Wong, L.S.Y., Hossain, S., Ta, A., Edvinsson, J., Rivas, D.H., and Naas, H. "A very low-power CMOS mixed-signal IC for implantable pacemaker applications", IEEE Journal of Solid-State Circuits, 39(12), pp. 2446-2456 (2004).

7. Martinez, J.S. and Suner, J.S. "IC voltage to current transducers with very small transconductance", Ana- 
log Integrated Circuits and Signal Processing, 13(3), pp. 285-293 (1997).

8. Rodriguez-Villegas, E., Low Power and Low Voltage Circuit Design with the FGMOS Transistor, The Institution of Engineering and Technology, UK, pp. 1-304 (2006).

9. Aggarwal, B. and Gupta, M. "Low-voltage bulkdriven class $\mathrm{AB}$ four quadrant CMOS current multiplier", Analog Integrated Circuits and Signal Processing, 65(1), pp. 163-169 (2010).

10. Raikos, G. and Vlassis, S. "0.8 V bulk-driven operational amplifier", Analog Integrated Circuits and Signal Processing, 63(3), pp. 425-432 (2010).

11. Khateb, F., Khatib, N., and Kubánek, D. "Novel lowvoltage low-power high-precision CCII based on bulkdriven folded cascode OTA", Microelectronics Journal, 42(5), pp. 622-631 (2011).

12. Carrillo, J.M., Torelli, G., Perrez-Aloe, R., Valverde, J.M., and Duque-Carrillo, J.F. "Single-pair bulkdriven CMOS input stage: a compact low-voltage analog cell for scaled technologies", Integration, The VLSI Journal, 43(3), pp. 251-257 (2010).

13. Urban, C., Moon, J.E., and Mukund, P.R. "Scaling the bulk-driven MOSFET into deca-nanometer bulk CMOS processes", Microelectronics Reliability, 51(4), pp. 727-732 (2011).

14. Carrillo, J.M., Perez-Aloe, R., Valverde, J.M., and Duque-Carrillo, J.F. "Compact low-voltage rail-to-rail bulk-driven CMOS opamp for scaled technologies", European Conference on Circuit Theory and Design, pp. 263-266 (2009).

15. Carrillo, J.M., Torelli, G., Perez-Aloe, R., and DuqueCarrillo, J.F. "1-V rail-to-rail CMOS OpAmp with improved bulk-driven input stage", IEEE Journal of Solid-State Circuits, 42(3), pp. 508-517 (2007).

16. Raikos, G. and Vlassis, S. "Low-voltage bulk-driven input stage with improved transconductance", International Journal of Circuit Theory and Applications, 39(3), pp. 327-339 (2011).

17. Gupta, M. and Pandey, R. "Low-voltage FGMOS based analog building blocks", Microelectronics Journal, 42(6), pp. 903-991 (2011).

18. Gupta, M. and Pandey, R. "FGMOS based voltagecontrolled resistor and its applications", Microelectronics Journal, 41(1), pp. 25-32 (2010).

19. Pandey, R. and Gupta, M. "FGMOS based tunable grounded resistor", Analog Integrated Circuits and Signal Processing, 65(3), pp. 437-443 (2010).

20. Madhushankara, M. and Kumar Shetty, P. "Floating gate Wilson current mirror for low power applications", Communications in Computer and Information Science, Springer, Berlin, 197, pp. 500-507 (2011).

21. Khateb, F., Khatib, N., and Koton, J. "Novel lowvoltage ultra-low-power DVCC based on floating-gate folded cascode OTA", Microelectronics Journal, 42(8), pp. 1010-1017 (2010).

22. Berg, Y., Næss, $\varnothing$, Høvin, M.E., and Gundersen, H. "Ultra low-voltage floating-gate (FGUVMOS) amplifiers", Analog Integrated Circuits and Signal Processing, 26(1), pp. 63-73 (2001).

23. Khateb, F., Khatib, N., and Kubánek, D. "Novel ultra-low-power class AB CCII+ based on floatinggate folded cascode OTA", Circuits Systems and Signal Processing, 31(2), pp. 447-464 (2012).

24. Thongleam, T., Suadet, A., and Kasemsuwan, V. "A $0.8 \mathrm{~V}$ quasi-floating-gate fully differential CMOS opamp with positive feedback", 8th International Conference on Electrical Engineering/Electronics, Computer, Telecommunications and Information Technology (ECTI-CON), pp. 98-101 (2011).

25. Moradzadeh, H. and Azhari, S.J. "High performance low-voltage QFG-based DVCC and a novel fully differential SC integrator based on it", IEICE Electronics Express, 5(23), pp. 1017-1023 (2008).

26. Khateb, F., Bay Abo Dabbous, S., and Vlassis, S. "A survey of non-conventional techniques for low-voltage low-power analog circuit design", Radioengineering J., 22(2), pp. 415-427 (2013).

27. Lopez-Martin, A.J., Angulo, J.R., Carvajal, R.G., and Acosta, L., "Micropower high current-drive class AB CMOS current-feedback operational amplifier", International Journal of Circuit Theory and Applications, 39(9), pp. 893-903 (2010).

28. Lopez-Martin, A.J., Acosta, L., Alberdi, C.G., Carvajal, R.G., and Angulo, J.R. "Power-efficient analog design based on the class AB super source follower", International Journal of Circuit Theory and Applications, 40(11), pp. 1143-1163 (2011).

29. Torralba, A., Lujan-Martinez, C., Carvajal, R.G., Galan, J., Pennisi, M., Angulo-Ramirez, J., and Lopez-Martin, A. "Tunable linear MOS resistors using quasi-floating-gate techniques", IEEE Transactions on Circuits and Systems II, 56(1), pp. 41-45 (2009).

30. Zhang, B.J., Yang, Y.T., and Zhang, H.J. "A fully balanced fifth-order low-pass Chebyshev filter based on quasi-floating gate transistors", IEEE Conference on Electron Devices and Solid-State Circuits, pp. 537540 (2005).

31. Gupta, R., Sharma, S., and Jamuar, S.S. "A low voltage current mirror based on quasi-floating gate MOSFETs", IEEE Asia Pacific Conference on Circuits and Systems, pp. 580-583 (2010).

32. Safari, L. and Azhari, S.J. "An ultra low power, low voltage tailless QFG based differential amplifier with high CMRR, rail to rail operation and enhanced slew rate", Analog Integrated Circuits and Signal Processing, 67(2), pp. 241-252 (2011).

33. Alberdi, C.G., Lopez-Martin,, A.J., Acosta, L., Carva- 
jal, R.G., and Ramirez-Angulo, J. "Class AB CMOS tunable transconductor", 53rd IEEE International Midwest Symposium on Circuits and Systems, pp. 596599 (2010).

34. Algueta Miguel, J.M., De La Cruz Blas, C.A. and Lopez-Martin, A.J. "CMOS triode transconductor based on quasi-floating-gate transistors", Electronics Letters, 46(17), pp. 1190-1191 (2010).

35. Miguel, J.M.A., Lopez-Martin, A.J., Acosta, L., Ramirez-Angulo, J., and Carvajal, R.G. "Using floating gate and quasi-floating gate techniques for railto-rail tunable CMOS transconductor design", IEEE Transactions on Circuits and Systems I, 58(7), pp. 1604-1614 (2011).

36. Calvo, B., Lopez-Martin, A.J., Balasubramanian, S., Ramirez-Angulo, J., and Carvajal, R.G. "Linearenhanced $\mathrm{V}$ to I converters based on MOS resistive source degeneration", IEEE International Symposium on Circuits and Systems, pp. 3118-3121 (2008).

37. Hassan, T.M. and Mahmoud, S.A. "New CMOS DVCC realization and applications to instrumentation amplifier and active-RC filters", $A E U$ - International Journal of Electronics and Communications, 64(1), pp. $47-55$ (2010).

38. Khan, I.A. and Beg, P. "Fully differential sinusoidal quadrature oscillator using CMOS DVCC", International Conference on Communication, Computer and Power, pp. 196-198 (2009).

39. Ibrahim, M.A., Minaei, S., and Kuntman, H. "DVCC based differential-mode all-pass and notch filters with high CMRR", International Journal of Electronics, 93(4), pp. 231-240 (2006).

40. Soliman, A.M. "Low voltage wide range CMOS differential voltage current conveyor and its applications", Contemporary Engineering Sciences, 1(3), pp. 105-126 (2008).

41. Naik, A.P. and Devashrayee, N.M. "Characterization of a CMOS differential current conveyor using 0.25 micron technology", International Journal of Advanced Engineering \& Applications, 23, pp. 177-182 (2010).

42. Chen, H.P. "Tunable versatile current-mode universal filter based on plus-type DVCCs", AEU - International Journal of Electronics and Communications, 66(4), pp. 332-339 (2012).

43. Chen, H.P. and Shen, S.S. "A versatile universal capacitor-grounded voltage-mode filter using DVCCs", ETRI Journal, 29(4), pp. 470-476 (2009).

44. Khateb, F., Khatib, N., and Kubánek, D. "Lowvoltage ultra-low-power current conveyor based on quasi-floating gate transistors", Radioengineering $J$., 21(2), pp. 725-735 (2012).

45. Minaei, S., Ibrahim, M.A., and Kuntman, H. "DVCC based current-mode first-order all-pass filter and its application", 10th IEEE International Conference on Electronics, Circuits and Systems, pp. 276-279 (2003).
46. Horowitz, P. and Hill, W. The Art of Electronics, Cambridge University Press, U.K., pp. 1-291 (1991).

47. Tietze, U. and Schenk, C.K., Electronic Circuits: Design and Applications, Springer, pp. 795-796 (1991).

48. Holzel, R. "A simple wide-band sine wave quadrature oscillator", IEEE Transactions on Instrumentation and Measurement, 42(3), pp. 758-760 (1993).

49. Khan, I.A. and Khwaja, S. "An Integrable gm-C quadrature oscillator", International Journal of Electronics, 87(11), pp. 1353-1357 (2000).

\section{Biographies}

Fabian Khateb received the $\mathrm{MSc}$ and $\mathrm{PhD}$ degrees in Electrical Engineering and Communication as well as in Business and Management from Brno University of Technology (BUT), Czech Republic, in 2002, 2005, 2003, and 2007, respectively. He is currently working as Associate Professor in the Department of Microelectronics at BUT. He has expertise in new principles of designing low-voltage low-power analog circuits, particularly in biomedical applications. He is author or co-author of more than 90 publications in journals and proceedings of international conferences.

Nabhan Khatib received the $\mathrm{MSc}$ and $\mathrm{PhD}$ degrees in Electrical Engineering and Communication from Brno University of Technology (BUT), Czech Republic, in 2009 and 2013, respectively. Currently, he is working as scientific researcher in the Department of Microelectronics at BUT. His scientific activities are directed towards the area of low-voltage low-power analog circuits for signal processing based on nonconventional active elements. He has been selected as reviewer in numerous scientific international journals. He is author or coauthor of more than 25 publications in journals and proceedings of international conferences.

Jaroslav Koton received the $\mathrm{MSc}$ and $\mathrm{PhD}$ degrees in Electrical Engineering from the Brno University of Technology (BUT), Brno, Czech Republic, in 2006 and 2009, respectively. Currently, he works as Associate Professor in the Department of Telecommunications at BUT. $\mathrm{He}$ is also active as a senior researcher and vice-head of Converged Systems Program of the SIX Research Centre, where his research and development activities are focused on linear and nonlinear circuit designing methods with current or voltage conveyors, and current active elements. $\mathrm{He}$ is an author or coauthor of about 131 research articles published in international journals or conference proceedings.

Norbert Herencsar received the $\mathrm{MSc}$ and $\mathrm{PhD}$ degrees in Electronics \& Communication and Telein- 
formatics from Brno University of Technology, Czech Republic, in 2006 and 2010, respectively. Currently, he is an Assistant Professor in the Department of Telecommunications and Research Fellow in the Centre of Sensor, Information and Communication Systems (SIX) of Faculty of Electrical Engineering and Com- munication, Brno University of Technology, Brno, Czech Republic. He is an author or co-author of 49 research articles published in SCI-E international journals, 23 articles published in other journals, and 78 papers published in proceedings of international conferences. 